\title{
A Case Report of a Node Positive Breast Implant Associated - Anaplastic Large Cell Lymphoma
}

\author{
Mustata $\mathrm{L}^{1 *}$, Collins G², Ilie D ${ }^{3}$, Parulekar V ${ }^{3}$, Adams R $^{3}$ and Roy PG ${ }^{4}$ \\ ${ }^{1}$ Breast Surgery Department, Oxford University Hospitals, UK; Gynecology Dpt. \\ Filantropia Clinical Hospital, Bucharest, Romania \\ ${ }^{2}$ Oncology Department, Oxford University Hospitals, UK \\ ${ }^{3}$ Breast Radiology Department, Oxford University Hospitals, UK \\ ${ }^{4}$ Breast Surgery Department, Oxford University Hospitals, UK
}

\section{Research Article \\ Volume 3 Issue 2}

Received Date: October 15, 2019

Published Date: November 08, 2019

DOI: $10.23880 /$ ijtps-16000138

*Corresponding author: Laura Mustata, Breast Surgery Department, Oxford University Hospitals, UK; Gynecology Dpt. Filantropia Clinical Hospital, Bucharest, Romania, Tel: +40740297218; Email: lauramustata@yahoo.com

\section{Abstract}

Background: Breast implant-associated anaplastic large cell lymphoma (BIA-ALCL) is a rare condition associated with breast implants.

Methods: This paper describes a case of node positive breast implant associated ALCL in a young woman who underwent cosmetic breast augmentation 11 years prior to this diagnosis.

Results: She was the first case of BIA-ALCL treated in our institution and emphasizes the importance of multidisciplinary team approach involving breast surgeons, hematologists, pathologists, breast radiologists and radiation oncologists. We have reviewed the available literature in this report. Although most cases of implant associated ALCL can be treated with surgery alone as they are localized, the spread beyond the implant cavity involves discussion about systemic treatment and the sequencing of the various therapies.

Conclusion: Given the prevalence of implants in the community worldwide, it is important to raise awareness amongst medical staff and educate the general population to seek medical attention early.

A unique case report of node positive BIA ALCL

Keywords: Anaplastic Large Cell Lymphoma (BIA-ALCL); Breast Implants; Breast Cancer; Chemotherapy; Breast Surgery

\section{Introduction}

Breast implant-associated anaplastic large cell lymphoma (BIA-ALCL) is a rare type of T-cell nonHodgkin lymphoma related to breast implants. 173 cases have been reported with both reconstructive and cosmetic implants so far [1-3]. The first case of ALCL was reported in 1997 with a textured McGhan saline-filled breast implant (Keech and Creech) presenting as $20 \mathrm{~mm}$ lesion on the implant capsule. Patient underwent surgery 


\section{International Journal of Transplantation \& Plastic Surgery}

involving capsulectomy and implant replacement followed by chemotherapy and radiotherapy, ensuring disease free interval for the subsequent years [4].

Although the etiology of BIA-ALCL is not completely elucidated, the possible theories include chronic inflammatory response, immune response, type of implant (smooth or textured), hereditary predisposition or bacteria biofilm. It is obvious that there is an important connection between implants and BIA ALCL regarding the etio-pathogenesis, but questions remain unanswered about this subject [5-7]. One of the plausible causes seems to be the immune response of the T-cells to some antigens in the biofilm of the implant [8]. A study in Australia showed an association between textured implants and an increased number of $\mathrm{T}$ cell lymphocytes when compared to smooth surface implants [6,7]. FDA reported 359 cases of BIA ALCL (including 9 deceased) and from 231 that had details about the surface of the implant, over $90 \%$ were associated with textured surface of the implants. Of 321 cases that had this information on the type of fill, 186 were silicone gel-filled and 126 were saline filled [8]. 37 reports have been published with 173 cases of BIA ALCL [4]. Some patients diagnosed with BIA ALCL had undergone multiple revisional surgeries and exchange of implants and all had at least one textured implant in their medical history. In 1986 the textured surface implants were available and WHO identified the ALCL in 1985. Probably there are some unrecognized cases before this era $[4,9]$.

Cohort study in New Zeeland and Australia showed higher incidence rates, estimated risk was between $1 / 1000-1 / 10000$ in women with textured implants, presuming that only textured implants cause this condition [9-11]. A case-controlled study done in the Netherlands on a population of 9 million women showed an insignificant lower risk (the absolute risk) of developing BIA ALCL in the general population, but for women with breast implants, it was confirmed as 18 times higher. The incidence rate was 1-3 cases/1.000.000 women/year in the latter study. The tendency was higher 10 years post-reconstruction and the mean age at diagnosis was around 50. Nevertheless, some cases of early and late onset have been cited in the literature $[12,13]$. US study from 2017 showed a rate of incidence of 203 in 100.000 .000 persons/year and a 67 times higher rate of BIA ALCL than primary ALCL of breast [14-16].

Most of the patients present with unilateral breast enlargement or new seroma collection near the implant on a localized area, usually more than 10 years after implant was placed (without any significance when comparing cosmetic versus reconstruction implants) [1517]. There are a few cases reported to have presented with bilateral symptoms, such as effusion between the implant and the capsule. Often patients complain of breast swelling due to a peri-implant fluid collection, new lump, skin inflammatory changes, capsular contracture (nonspecific symptom, $10 \%$ of implants), pain, asymmetry $[1,17]$. Some reported skin lesion as dominant symptom, as papules and some generic clinical features as fever, fatigue, night sweats, axillary lymphadenopathy or other palpable nodes [17].

Guidelines for diagnosis and treatment of BIA ALCL were given by NCCN based on the cases that were reported. BIA ALCL should be suspected in cases of sudden seroma and breast swelling in patients with breast implants, at least one year after their breast surgery, after excluding any breast trauma or infection. Clinical examination should include breasts, axillae, neck and chest wall. If suspicion is raised, further imaging assessment is needed, and ultrasound should be the first approach with fluid aspiration for cytology analysis as gold standard diagnostic method. CT and MRI examination have the same rate of detection as ultrasound, thus ultrasound is the most used method (being cost efficient and accurate). PET CT should be performed to rule out systemic ALCL and for follow up $[3,17]$. Cytology is used for confirmation of diagnosis and immunohistochemistry analysis together with flow cytometry confirms T cell population, CD30 positive and large anaplastic cells [10].

Ultrasound examination can identify seroma, mass on the implant capsule or mass effect on the surrounding structures or thickened capsule. It is not recommended to routinely perform ultrasound guided aspiration of periimplant fluid to rule out ALCL; it is only for diagnostic purpose in suspected cases, performed by interventional radiologist or by clinicians using a blunt needle to avoid implant injury and leakage. The analysis of the fluid sample should be performed by a haemato-pathologist and cytology analysis, flow cytometry and immunohistochemistry will show the features regarding ALK, CD30 status and presence or anaplastic cells $[1,17]$.

The differential diagnosis includes primary or recurrent breast cancer, primary breast lymphoma, other forms of ALCL, infection, benign seroma, other breast implant complications [1]. 


\section{International Journal of Transplantation \& Plastic Surgery}

Staging for BIA ALCL is not clearly defined, whether it should follow TNM or Lymphoma guidelines; Ann Arbour system is used as well (early BIA ALCL limited on the breast - IE or limited on the breast and axilla - IIE). Following the natural history of BIA ALCL, this entity seems to metastasize as solid tumors and TNM staging is more suitable, being also useful for prognosis and oncological treatment $[1,3]$.

The staging is summarized in Table with Classification TNM VS Ann Arbour (TABLE 1).

\begin{tabular}{|c|c|c|c|}
\hline T - extent of the tumor & $\mathbf{N}$ - lymph nodes involvement & M - metastastis & \\
\hline $\begin{array}{c}\text { T1 - limited on the luminal side of } \\
\text { capsule }\end{array}$ & N0 - no lymph nodes involvement & M0 - no distant spread & \\
\hline T2 - limited capsule infiltration & N1 - one regional lymph node involved & M1 - distant spread & \\
\hline $\begin{array}{c}\text { T3 - cell aggregates infiltrationg } \\
\text { capsule }\end{array}$ & N2 - multiple nodes involved & & \\
\hline $\begin{array}{c}\text { T4 - disease infiltrates beyond } \\
\text { capsule }\end{array}$ & & & \\
\hline Ann Arbour & IIA T4N0M0 & III T4N1M0 & IV T1-4N1-2M1 \\
\hline IA T1N0M0 & IIB T1-3N1M0 & & \\
\hline IB T2N0M0 & & & \\
\hline IC T3N0M0 & & & \\
\hline
\end{tabular}

Table 1: Classification TNM Vs Ann Arbour from Clemens, et al. [25].

\section{Case Report}

We report a case of a 48-year-old Caucasian female with a history of bilateral cosmetic sub-muscular augmentation 11 years prior to this diagnosis. The implants used were Allergan textured, round, silicone implants (295cc; type ST110). The surgery was uneventful, and the operation details were available from the hospital notes. She presented with a 6 weeks history of left breast lump in august 2017. She has a medical history of right breast fibroadenoma that was biopsied in the past but not operated. With regards to her family history, her mother had breast cancer at age 65 . She had 2 children and didn't breast feed. She used contraceptive pill (mini-pill) for more than 5 years and she was premenopausal at the time of presentation.

On clinical examination at presentation, there was a large lump in the upper-inner quadrant of the left breast, with no obvious sign of implant rupture on either side. The presence of focal asymmetrical lump was not typical of capsular contracture. Imaging was performed; mammogram showed no abnormality; ultrasound of the right breast confirmed the known right breast fibroadenoma whilst there were multiple masses in the left breast in the upper inner quadrant raising suspicion of ALCL; there was no associated seroma or fluid collection. An ultrasound guided biopsy was performed. MRI described an ill-defined lobulated mass measuring $46 \times 35 \times 40 \mathrm{~mm}$, in the upper inner quadrant anterior and superior to the implant capsule, attached to the muscle. A small volume of fluid around the implant was reported. PET CT examination confirmed $6-7 \mathrm{~cm}$ irregular mass adjacent to the supero-medial aspect of left breast implant with a cluster of small left axillary nodes and a small suspicious left internal mammary node. There was no evidence of disease elsewhere in the body. A core biopsy was performed from the left axillary lymph nodes.

Histology revealed focally highly pleomorphic infiltrate of neoplastic large cells, with prominent nucleoli; multiple mitoses were seen; background with small lymphocytes and numerous eosinophils; IHC confirmed strongly positive for CD30 and CD45, positive for CD2, CD4, CD5 and GranzymeB; CD3, CD7, CD5 and CD8 was largely confined to a background of small reactive T-cells. The neoplastic cells were ALK-1, AE1/3, CD56 and CK7 negative; MIB1 proliferation index was $80 \%$.

Biopsy of the left axillary lymph nodes confirmed involvement of the lymph nodes. The final pathology was consistent with ALK-negative BIA ALCL with axillary lymph nodes involvement (stage IIB). The management was discussed in a multidisciplinary team including haematologists, oncologists, breast surgeons, hematology pathologist, breast pathologist and radiologist.

Pre-operative assessment of the disease showed potential unresectable disease, in discussion with thoracic 


\section{International Journal of Transplantation \& Plastic Surgery}

surgeon. Therefore, neoadjuvant chemotherapy was recommended with 6 courses of 2-weekly cyclophosphamide, doxorubicin, vincristine and prednisone (CHOP), followed by surgery and consolidation radiotherapy to the chest wall. PET CT performed post neoadjuvant chemotherapy showed complete radiological response in breast and lymph nodes, including internal mammary lymph node.

Surgery was performed consisting in bilateral removal of implants and capsulectomies under general anesthesia. Intraoperatively there was no residual mass/fluid collection found. The implant was removed "en masse" with the capsule. There was no obvious infiltration of the muscle. Contralateral surgery included right breast implant removal and capsulectomy. On macroscopic examination the right breast implant capsule was thinner, with no abnormality. No surgery was performed on the left axillary lymph nodes, given complete radiological response on PET-CT. The microscopic examination showed no evidence of malignancy on the left implant or capsule. The surgery was uneventful with no postoperative complications. Consolidation radiotherapy for the chest wall was recommended by the multidisciplinary team. The patient completed radiotherapy and she is planned to be reviewed every 3 to 6 months by the Haemato-oncologist for the next 5 years. The case was reported to the MHRA as per national guidance.

\section{Discussion}

The worldwide literature has reported few hundreds of cases so far and the reported incidence varies from $1 / 1000$ and $1-3 / 1000000[18,19]$. The majority present with intracapsular disease and are treated with surgery alone. The pathology and immunohistochemistry findings confirm ALK negative and CD30 positive BIA ALCL; these cases are distinct from ALK negative systemic ALCL. The treatment usually consists of surgery [18]. The majority of BIA ALCL were diagnosed early and treated with surgery alone. Few advanced cases were reported in the literature and several deaths were registered. However, there is no recommendation for screening or prophylactic removal of breast implants for asymptomatic patients irrespective of family history or genetic predisposition for breast cancer [1].

The possible etiologic entities that have been proposed include chronic inflammation in response to a foreign body, genetic susceptibility as gene mutation, gene dysplasia and gene degeneration, microbiology theory including unusual load of specific bacteria in the implant biofilm [1,19-23].

The cases reported so far demonstrated clinical presentation as seroma in $70 \%$ of cases, $30 \%$ as palpable mass and $10-17 \%$ had axillary lymphadenopathy corresponding to the side of the symptoms. Axillary metastasis was reported in $14,9 \%$ of cases. Usual laboratory investigations show unremarkable and nonspecific features (isolated eosinophilia) [1]. Imaging investigations include ultrasound and MRI; the routine investigation is ultrasound, being useful for guidance in fluid aspiration for diagnosis. PET CT is the recommended investigation for staging and evaluation of systemic extent of the disease. Mammogram examination brings poor information on BIA ALCL [1,17]. The final diagnosis is based on immunohistochemistry analysis, BIA ALCL being ALK negative, CD30 positive and epithelial surface antigen positive. T cell antigens CD4 and CD43 are expressed in up to $85 \%$ of the cases; CD3, CD2 and CD45 are positive in around $30-35 \%$ of the cases and rarely there is expression of CD5, CD7, CD15, CD8 [1,5,12]. Evaluation, treatment and management of BIA ALCL should be decided by a multidisciplinary team, with experts familiar with this medical condition. NCCN guidelines are uncertain and were decided from series of case reports due to the rarity of this pathological entity and prospective studies are to follow $[1,3]$.

Few cases of spontaneous remission for seroma have been cited in the literature with clinical surveillance $[16,24]$. Primary treatment includes surgical removal of the mass, the implant and capsulectomy and the aim is to acquire clear margins for oncology safety. If complete resection is achieved, no further adjuvant treatment is required $[1,25]$. Surgery should be performed guided by general oncology principles with orientation and marking of the specimen, marker clips in the remaining cavity. Guidelines suggest removing contralateral implant with capsule to prevent recurrent disease or lymphoma on the other breast $[25,26]$.

Surgery to axilla needs not be performed routinely in the absence of evidence of nodal involvement. For cases with node enlargement, excision biopsy should be performed to confirm the pathology. More than a half of the patients with abnormal nodes showed metastatic disease in the reported literature $[17,25]$.

The current NCCN guidelines recommend neoadjuvant chemotherapy with lymphoma regimes for mass presentation and locally advanced disease with lymph- 


\section{International Journal of Transplantation \& Plastic Surgery}

node involvement $[1,3,25]$. A retrospective study that included 87 patients with BIA ALCL compared surgery with chemo/radiotherapy approach and the disease-free interval in 3 years follow up was longer for patients who underwent surgery with complete resection. Adjuvant chemotherapy and radiotherapy is not usually recommended in cases of early disease and in cases where complete surgical removal is achieved $[1,11]$.

Chemotherapy and radiotherapy as adjuvant therapy for incomplete resection had lower rates of survival and disease-free interval. The reported rate of recurrence in these cases is between 18 and 24\% [1,24]. Oncology case reports suggest that Brentuximab regimes used for refractory disease can achieve complete remission and it is recommended as a first line treatment when chemotherapy needs to be avoided for toxicity reasons [18].

There is still a debate regarding axillary clearance in cases with neoadjuvant chemotherapy and possible benefit apart from removal of the implant and capsulectomy. The current indication for lymph-node removal is controversial and if complete radiological response has been achieved with CHOP regimes, then radiotherapy could be considered as adjuvant therapy to prevent recurrence, instead of axillary clearance. For women with nodal disease, a more aggressive approach is suggested, due to higher risk of recurrence $[10,25]$.

A retrospective study reported 55 patients who underwent surgery for BIA ALCL [26]. 39 had adjuvant chemotherapy, 12 patients had watchful waiting and 4 patient had adjuvant radiotherapy. The outcomes were similar, and the survival rate and disease-free interval were higher for patients who had surgery with or without adjuvant chemotherapy. A comparison between patients presenting with a mass and those presenting with seroma showed that the survival rate and disease-free interval were higher for seroma cases. Patients presenting with breast mass who underwent complete resection of the tumor had similar rates of recurrence and survival rate or disease-free interval when compared to those with seroma presentation $[2,18,25-27]$.

There are a few cases treated with radiotherapy alone and most of them were locally advanced $[1,17,25]$. Implant replacement remains controversial and it is not recommended, at least not with textured implants, but there were cited few cases of implant replacement with smooth textured implants. A close follow-up is recommended for those patients [1,17]. BIA ALCL patients should be followed up clinically every 3-6 months for the first 5 years in a multidisciplinary team and annual ultrasound for the first two years. There are some reports suggesting CT thorax, abdomen and pelvis or PET CT every 6 months for the first 2 years $[1,17,27]$.

Adjuvant chemotherapy or radiotherapy is recommended for disseminated disease, recurrent or incomplete surgical resection. Anthracycline chemotherapy regimens and immunotherapy with anti CD30 drug (Brentuximab) may be recommended as primary approach, however there is need for more data and multiple trials are ongoing. Regarding radiotherapy, the advantage of radiation therapy for primary cutaneous ALCL is well known, but the benefits are yet to be demonstrated for BIA ALCL. Radiotherapy alone has not been shown as efficient when complete resection is achievable $[1,17,25,27]$.

Moreover, taking into consideration that worldwide there are 35 million patients with breast implants and only few hundred cases of BIA ALCL have been reported, there is no recommendation from any medical regulation society to remove the breast implants as preventative policy. Although the textured implants have been in the middle of the crisis related to the BIA ALCL and a few deaths were recorded related with this diagnosis, the risks and benefits of these medical devices are well known and acknowledged. Implant registry should be the next step in all countries using breast implants in order to record all patients and implants that are being used [28].

Most discussed risk factor for BIA ALCL still is the textured implants. Surgical technique, percentage of capsular contracture, strategies to improve sterility and operating time were demonstrated as no reliable factors to reduce the BIA ALCL risk. The constant of this discussion remains the textured implants. Few analyses were done recently and demonstrated that Biocell textured implants have the highest risk of BIA ALCL and FDA removed them as medical devices. Patients' groups and other medical professional groups are debating removal of textured breast implants as medical device, because no change in surgical technique or other modifiable factor from the implant pathway was demonstrated efficient to prevent BIA ALCL [29].

Research is being done currently to establish the clear pathogenesis of the disease. A possible theory includes an exacerbated immune response to the inflammatory microenvironment that releases cytokines and chemokines in the area and this pathway leads to seroma 


\section{International Journal of Transplantation \& Plastic Surgery}

formation. Mutation of JAK STAT 3 leads further to T cell proliferation. Although this mutation was found only in few cases, targeted therapy for JAK proteins warrant further research for the BIA ALCL and understanding of the pathogenesis [30]. Other pathogenesis pathway studied included lately the gram-negative microbiome, therefor use of antiseptic solution with broad spectrum tried to reduce this risk by irrigating the breast pocket. The pocket was prepared with both betadine and nonbetadine solution and the betadine alone showed superior effect than others with higher bactericidal effect and improving inflammation [31].

In the available literature, complete surgical resection has the best outcome ( $4 \%$ risk of recurrence), when achieved. Poor prognosis includes bilateral disease, extracapsular extension of disease or mass presentation. $[1,25,32]$.

\section{Conclusion}

In the case reported we wish to highlight the role of neoadjuvant chemotherapy in node positive and potentially unresectable disease, as primary surgery could have resulted in incomplete resection, potentially altering the prognosis.

It is also important to highlight the multidisciplinary approach with hematology and breast team, including surgeons, pathologists, radiologist and oncologist.

Currently patients in UK are being reported to MHRA as per indicated guidance (with similar registry in other countries) and this will help unify the data and provide insight from cases worldwide.

Although BIA ALCL is a very rare condition and the pathogenesis is not fully understood yet, early diagnosis and prompt surgical and oncological approach can be lifesaving for patients. The presentation with seroma around breast implants could potentially result in decision to revisional cosmetic surgery in the absence of adequate work-up. Therefore it is important to raise awareness and any swelling/ firm lump to be investigated prior to undertaking revisional surgery. Patients with breast implants, clinicians, and specialists in primary care should be trained to recognize and to act efficiently and responsibly if any suspicion $[1,2,32]$.

\section{Additional Information Section}

Ethical approval and consent to participate: A local ethics committee at the Oxford University Hospital ruled that no formal ethics approval was required in this particular case. The case was formally reported to MHRA as per national guidance.

Consent to publish: Patient has consented for anonymized data to be presented and published. Data Availability.

All medical data are provided in the manuscript.

Conflict of interest: The authors declared no potential conflicts of interest with respect to the research, authorship, and publication of this article.

Funding: The authors received no financial support for the research, authorship, and publication of this article.

The authors declare that the study was performed in accordance with the Declaration of Helsinki.

\section{References}

1. Clemens MW, Jacobsen E, Butler CE, Freedman AS (2017) Breast implant-associated anaplastic large cell lymphoma.

2. O'Neill AC, Zhong T, Hofer SOP (2017) Implications of Breast Implant-Associated Anaplastic Large Cell Lymphoma (BIA-ALCL) for Breast Cancer Reconstruction: An Update for Surgical Oncologists. Ann Surg Oncol 24(11): 3174-3179.

3. Clemens MW, Horwitz SM (2017) NCCN Consensus Guidelines for the Diagnosis and Management of Breast Implant-Associated Anaplastic Large Cell Lymphoma. Aesthet Surg J 37(3): 285-289.

4. Brody GS, Deapen D, Taylor CR, Pinter-Brown L, House-Lightner SR, et al. (2015) Anaplastic large cell lymphoma occurring in women with breast implants: analysis of 173 cases. Plast Reconstr Surg 135(3): 695-705.

5. Ferreri AJ, Govi S, Pileri SA, Savage KJ (2013) Anaplastic large cell lymphoma, ALK-negative. Crit Rev Oncol Hematol 85(2): 206-215.

6. Jacombs A, Tahir S, Hu H, Deva AK, Almatroudi A, et al. (2014) In vitro and in vivo investigation of the influence of implant surface on the formation of bacterial biofilm in mammary implants. Plast Reconstr Surg 133(4): 471e-80e.

7. Hu H, Jacombs A, Vickery K, Merten SL, Pennington DG, et al. (2015) Chronic biofilm infection in breast implants is associated with an increased T-cell 


\section{International Journal of Transplantation \& Plastic Surgery}

lymphocytic infiltrate: implications for breast implant-associated lymphoma. Plast Reconstr Surg 135(2): 319-329.

8. Medical Device Reports of Breast Implant Associated Anaplastic Large Cell Lymphoma.

9. Loch-Wilkinson A, Beath KJ, Knight RJW, Wessels WLF, Magnusson M, et al. (2017) Breast ImplantAssociated Anaplastic Large Cell Lymphoma in Australia and New Zealand: High Surface Area Textured implants are associated with increased Risk. Plast Reconstr Surg 140(4): 645-654.

10. Ezekwudo DE, Ifabiyi T, Gbadamosi B, Haberichter K, $\mathrm{Yu}$ Z, et al. (2017) Breast Implant-Associated Anaplastic Large Cell Lymphoma: A Case Report and Review of the Literature. Case Reports Oncological Med 2017: 6.

11. Lazzeri D, Agostini T, Bocci G, Giannotti G, Fanelli G, et al. (2011) ALK-1-negative anaplastic large cell lymphoma associated with breast implants: a new clinical entity. Clin Breast Cancer 11(5): 283-296.

12. de Jong D, Vasmel WL, de Boer JP, Verhave G, Barbé E, et al. (2008) Anaplastic large-cell lymphoma in women with breast implants. JAMA 300(17): 20302035.

13. Doren EL, Miranda RN, Selber JC, Garvey PB, Liu J, et al. (2017) U.S. Epidemiology of Breast ImplantAssociated Anaplastic Large Cell Lymphoma. Plast Reconstr Surg 139(5): 1042-1050.

14. Miranda RN, Aladily TN, Prince HM, KanagalShamanna R, de Jong D, et al. (2014) Breast implantassociated anaplastic large-cell lymphoma: long-term follow-up of 60 patients. J Clin Oncol 32(2): 114-120.

15. Albornoz CR, Bach PB, Mehrara BJ, Disa JJ, Pusic AL, et al. (2013) A paradigm shift in U.S. Breast reconstruction: increasing implant rates. Plast Reconstr Surg 131(1): 15-23.

16. Leberfinger AN, Behar BJ, Williams NC, Rakszawski KL, Potochny JD, et al. (2017) Breast ImplantAssociated Anaplastic Large Cell Lymphoma A Systematic Review. JAMA Surg 152(12): 1161-1168.

17. Richardson L (2017) Breast implant associated anaplastic large cell lymphoma -case study. Molecular Clin Oncol 6: 539-542.
18. George EV, Pharm J, Houston C, Al-Quran S, Brian G, et al. (2013) Breast implant-associated ALK-negative anaplastic large cell lymphoma: a case report and discussion of possible pathogenesis. Int J Clin Exp Pathol 6(8): 1631-1642.

19. Bizjak M, Selmi C, Praprotnik S, Bruck O, Perricone C, et al. (2015) Silicone implants and lymphoma: The role of inflammation. J Autoimmun 65: 64-73.

20. Orciani M, Sorgentoni G, Torresetti M, Di Primio R, Di Benedetto G (2016) MSCs and inflammation: new insights into the potential association between ALCL and breast implants. Breast Cancer Res Treat 156(1): 65-72.

21. Hu H, Johani K, Almatroudi A, Vickery K, Van Natta B, et al. (2016) Bacterial Biofilm Infection Detected in Breast Implant-Associated Anaplastic Large-Cell Lymphoma. Plast Reconstr Surg 137(6): 1659-1669.

22. Adams WP (2016) Discussion: Bacterial Biofilm Infection Detected in Breast Implant-Associated Anaplastic Large-Cell Lymphoma. Plast Reconstr Surg 137(6): 1670-1672.

23. Fleming D, Stone J, Tansley P (2018) Spontaneous Regression and Resolution of Breast ImplantAssociated Anaplastic Large Cell Lymphoma: Implications for Research, Diagnosis and Clinical Management. Aesth Plast Surg 42(3): 672-678.

24. Clemens MW, Medeiros LJ, Butler CE, Hunt KK, Fanale MA, et al. (2016) Complete surgical excision is essential for the management of patients with breast implant-associated anaplastic large-cell lymphoma. J Clin Oncol 34(2): 160-168.

25. Kim B, Roth C, Chung KC, Young VL, van Busum K, et al. (2011) Anaplastic large cell lymphoma and breast implants: a systematic review. Plast Reconstr Surg 127(6): 2141-2150.

26. Clemens MW, Nava MB, Rocco N, Miranda RN (2017) Understanding rare adverse sequelae of breast implants: anaplastic large-cell lymphoma, late seromas, and double capsules. Gland Surg 6(2): 169184.

27. K Groth A, Graf R (2019) Breast Implant-Associated Anaplastic Large Cell Lymphoma (BIA-ALCL) and the Textured Breast Implant Crisis. Aesthetic Plast Surg. 


\section{International Journal of Transplantation \& Plastic Surgery}

28. Swanson E (2019) Plastic Surgeons Defend Textured Breast Implants at 2019 U.S. Food and Drug Administration Hearing: Why It Is Time to Reconsider. Plast Reconstr Surg Glob Open 7(8): e2410.

29. Turner SD, Inghirami G, Miranda $\mathrm{RN}$, Kadin $\mathrm{ME}$ (2019) Cell of Origin and Immunological Events in the Pathogenesis of Breast Implant-Associated Anaplastic Large Cell Lymphoma. Am J Pathol S0002-9440 (19): 30753-30759.

30. Culbertson EJ, Felder-Scott C, Deva AK, Greenberg DE, Adams WP (2019) Optimizing Breast Pocket Irrigation: The Breast Implant-Associated Anaplastic
Large Cell Lymphoma (BIA-ALCL) Era. Aesthet Surg J : sjz246.

31. Gardani M, Bellini E, Villani G, Orsi N, Palli D (2019) Breast implant-associated anaplastic large cell lymphoma: A rare case report of lymphoma in the form of a pericapsular solid formation. Breast J.

32. de Oliveira Sermoud LMC, Romano S, Chveid M, da Silva Amorim GL (2019) Breast Implant-Associated Anaplastic Large-Cell Lymphoma: Why Must We Learn About It? J Glob Oncol 5: 1-5. 